\title{
Análise do Processo de Fragmentação da Floresta nos Municípios de Corumbiara e Buritis - RO
}

\author{
Olívia Bueno Costa ${ }^{1}$, Eraldo Aparecido Trondoli Matricardi' ${ }^{1}$, \\ José Salatiel Rodrigues Pires ${ }^{2}$
}

\author{
${ }^{1}$ Departamento de Engenharia Florestal, Universidade de Brasília - UnB, Brasília/DF, Brasil \\ ${ }^{2}$ Departamento de Ciências Ambientais, Universidade Federal de São Carlos - UFSCar, São Carlos/SP, Brasil
}

\section{RESUMO}

A expansão das áreas de pastagens e de produção de grãos no estado de Rondônia se deu de forma acelerada na última década devido principalmente a incentivos fiscais e a alta demanda do mercado externo. Em consequência, graves processos de degradação da floresta se iniciaram. Este estudo objetivou analisar processos de fragmentação da floresta nos municípios de Corumbiara e Buritis, estado de Rondônia. Para tanto, foram utilizadas imagens Landsat 5 TM para classificar as áreas de floresta e não floresta. A avaliação da acurácia para a classificação supervisionada Maximum Likelihood indicou acurácia global de 91\% e Kappa de 78\%. Os resultados do cálculo de métricas indicam um aumento do retalhamento da paisagem ao longo da última década nos municípios, com aumento do efeito de borda, diminuição significativa da área da classe floresta e diminuição da conectividade das manchas de floresta. Assim, com base neste estudo, pôde ser observado um processo contínuo de aumento da fragmentação da floresta nativa nesses municípios.

Palavras-chave: sensoriamento remoto, análise de paisagem, mudança no uso da terra.

\section{Forest Fragmentation Analysis in the Municipalities of Corumbiara and Buritis, Rondônia State, Brazil}

\begin{abstract}
The expansion of agriculture and pasture in Rondônia state was accelerated in the past decade due to fiscal incentives and higher external market demand. Consequently, serious forest degradation processes were initiated. The present study aims to analyze forest fragmentation in the municipalities of Corumbiara and Buritis. To this end, Landsat 5 TM images were used for forest and non-forest classification. The accuracy assessment for Maximum Likelihood supervised classification indicated an overall accuracy of $91 \%$ and Kappa of $78 \%$. Results indicated an increase in the number of patches in the area of study in the past decade, with an increase in edge effects, significant reduction of forest class area, and a decrease in the connectivity of forest patches. Therefore, based on this study, a continuous increase in the fragmentation of native vegetation in these municipalities can be observed.
\end{abstract}

Keywords: remote sensing, landscape analysis, land-use changes. 


\section{INTRODUÇÃO}

O estado de Rondônia é marcado por intensos processos de ocupação e conversão de uso da terra desde a década de 1970 (Becker et al., 1990; Schmink \& Wood, 1992; Perdigão \& Bassegio, 1992; Matos et al., 2011). Esses processos de ocupação se intensificaram com os projetos de colonização orientados pelo governo federal com apoio do Instituto Nacional de Colonização e Reforma Agrária (Incra). Em consequência, milhares de imigrantes assentaram-se nas terras distribuídas do projeto, resultando em grande perda de floresta nativa (Skole \& Tucker, 1993; Malingreau \& Tucker, 1988; Bunker, 1999). Isso ocasionou um grave processo de fragmentação (Pedlowski et al., 1997), que segue até hoje. Atualmente, Rondônia apresenta $35,1 \%$ de seu território desmatado, o que corresponde a $83.389 \mathrm{~km}^{2}$ em área (INPE, 2015). Esse desmatamento se dá principalmente às margens da Rodovia BR-364 (De Barros Ferraz et al., 2005), pela qual ocorre o escoamento da produção do estado. Processos de fragmentação da Amazônia são frequentemente relatados na literatura (Skole \& Tucker, 1993; Arima et al., 2008; Broadbent et al., 2008; Briant et al., 2010; Laurance et al., 2011; Numata \& Cochrane, 2012) observando os graves efeitos de perda de biodiversidade desse complexo e importante bioma.

As principais transformações no uso da terra em Rondônia são ocasionadas pela expansão do pasto e, mais recentemente da soja, (Fearnside, 2001), que tem acelerado os processos de fragmentação e de perda de floresta no estado de Rondônia (De Barros Ferraz et al., 2005; Ferraz et al. 2006; Nepstad et al., 2002). A soja ocupa principalmente os municípios ao sul de Rondônia, que juntos apresentaram área plantada de quase 140 mil hectares no ano de 2011 (CONAB, 2012). A expansão desses plantios é impulsionada pela demanda do mercado externo para alimentação animal e humana aliada aos altos preços que a soja tem atingido no mercado externo que, segundo dados da CONAB (2012), chegaram a 25 reais cada saca (equivalente a $60 \mathrm{~kg}$ de grãos de soja).

Nesse cenário de expansão e mudanças no uso da terra, os processos de fragmentação são frequentes. Os efeitos ambientais da fragmentação incluem a eliminação de espécies, redução das populações e ainda a redução no tamanho e conectividade dos ambientes (Shida \& Pivello, 2005; Collinge, 1996).
O desenvolvimento econômico também deve ser considerado, pois faz com que a floresta se torne mais fragmentada e o risco de um incêndio aumente, já que aumenta o número de bordas suscetíveis que serão expostas mais frequentemente ao fogo (Cochrane et al., 2002; Cochrane \& Laurance, 2002). Na Amazônia esse risco é ainda maior, uma vez que florestas fragmentadas estão rodeadas de pastos e áreas que passam por queimadas periódicas, o que aumenta as fontes de ignição do fogo (Laurance \& Curran, 2008).

Corumbiara e Buritis são municípios do estado de Rondônia que apresentam grande parte de seu território desmatado e com graves processos de fragmentação. Corumbiara, área mais antiga de colonização, possui grande parte de seu território coberta de pastagens e culturas, como soja e cana, restando floresta nativa que ainda sofre processos de degradação. Já o município de Buritis, área de ocupação mais recente, sofreu processos mais intensos de degradação a partir de 2000. Esses processos se intensificaram em 2005 e continuam atuando até hoje. Esses dois municípios ainda sofrem alteração das paisagens e merecem um melhor entendimento acerca dessas mudanças. Assim, o uso de métodos de análise espacial para avaliar esses processos de fragmentação é fundamental em Rondônia (Batistella et al., 2000; Colson et al., 2009).

Baseado no exposto, o objetivo deste estudo consistiu na análise dos processos de fragmentação da floresta por meio de técnicas de sensoriamento remoto orbital nos municípios de Corumbiara e Buritis.

\section{METODOLOGIA}

O estudo da fragmentação da floresta foi realizado no município de Corumbiara e Buritis com enfoque nos anos de 2000, 2005 e 2011. Corumbiara localiza-se na porção sul de Rondônia; Buritis localiza-se na porção norte de Rondônia (Figura 1). Esses municípios foram escolhidos pelo alto grau de ocupação antrópica ao longo do tempo, com predominância de pasto e de plantios de soja, combinados com fragmentos remanescentes de floresta.

A análise de acurácia da classificação foi avaliada a partir de uma imagem SPOT 5 ( 9 de agosto de 2008) com 2,5 metros de resolução espacial e a imagem classificada, cena 230/069 do satélite Landsat sensor TM de 28 de junho de 2008. A partir do índice Kappa 


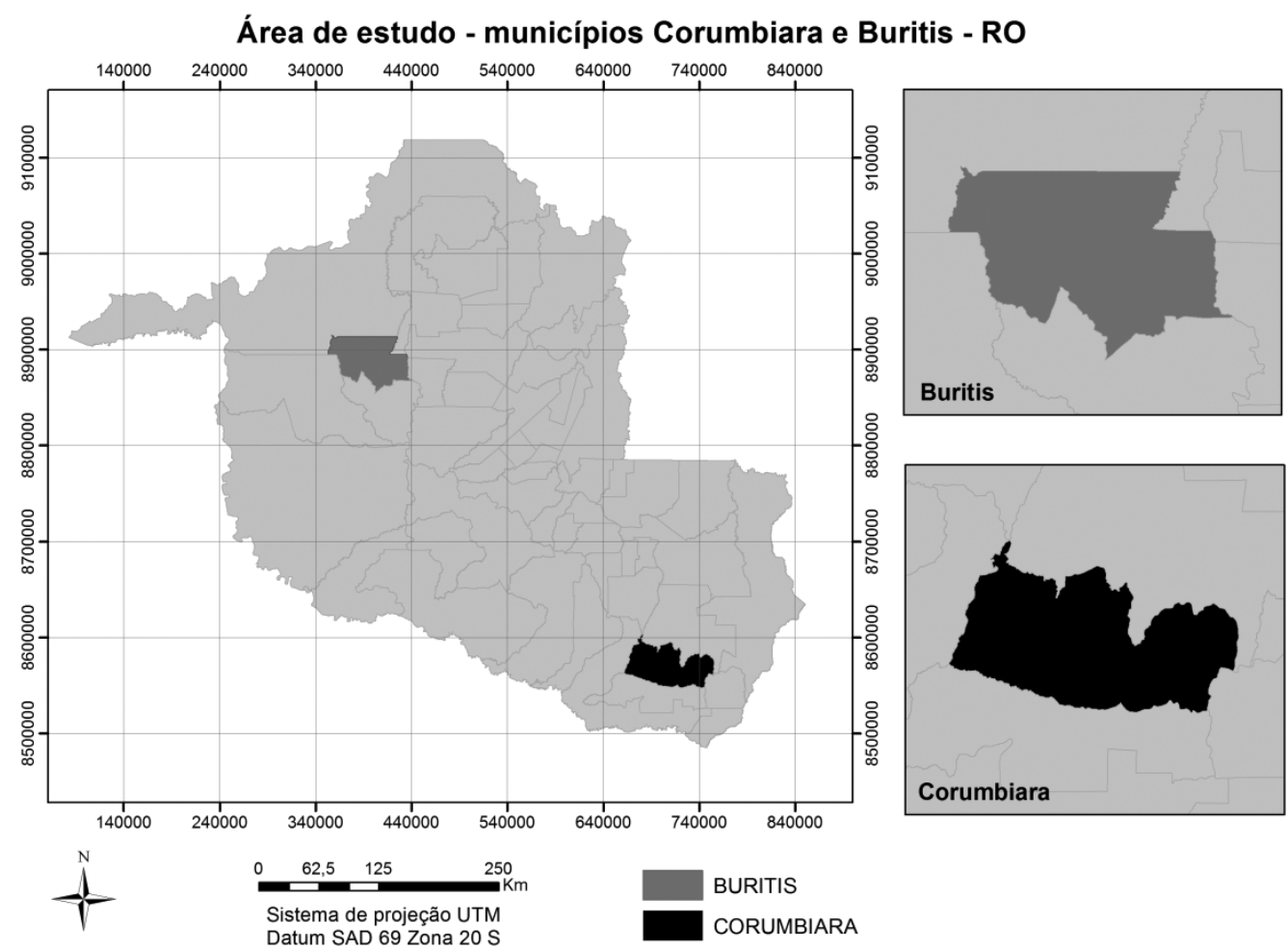

Figura 1. Municípios de Buritis e Corumbiara.

Figure 1. Corumbiara and Buritis municipalities.

(Cohen, 1960; Congalton, 1991) (Equação 1), a acurácia da classificação foi avaliada.

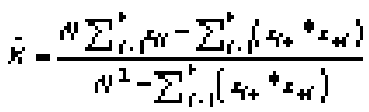

em que:= Índice de exatidão Kappa; $r$ = número de linhas na matriz; $x_{\mathrm{ii}}=$ número de observações na linha[i] e coluna[i]; $x_{i}+$ e $x_{+i}=$ totais marginais da linha[i] e coluna[i]; $\mathrm{N}=$ número total de observações.

Três cenas Landsat 5 TM (bandas 1-5 e 7) dos anos 2000, 2005 e 2011 (Tabela 1) foram utilizadas para o mapeamento do uso da terra na área de estudo.

A primeira etapa consistiu na calibração radiométrica que foi efetuada por meio da transformação de valores de números digitais das imagens Landsat 5 TM para valores de radiância no topo da atmosfera. Posteriormente efetuou-se a etapa de cálculo da reflectância, considerando-se os parâmetros disponibilizados no sítio do USDI (U.S. Department of the Interior, 2012) e de acordo com a metodologia proposta por Chander et al. (2009).
Tabela 1. Órbita, ponto e data das imagens utilizadas no estudo.

Table 1. Path, row and date of images used in this study.

\begin{tabular}{|cr|}
\hline Órbita/ponto & \multicolumn{1}{c|}{ Data } \\
\hline $230 / 069$ & 8 de julho de 2000 \\
\hline $232 / 067$ & 6 de julho de 2000 \\
\hline $230 / 069$ & 4 de junho de 2005 \\
$232 / 067$ & 2 de junho de 2005 \\
\hline $230 / 069$ & 21 de junho de 2011 \\
\hline $232 / 067$ & 5 de julho de 2011 \\
\hline
\end{tabular}

A correção geométrica foi feita a partir de imagens ortorretificadas do sítio da Universidade de Maryland (University of Maryland, 2012). As imagens Landsat 5 TM dos anos 2000, 2005, 2008 e 2011 foram georreferenciadas no sistema de projeção UTM, zona 20S e Datum SAD 69 no software ERDAS 9.1. O registro foi feito a partir do polinômio de primeira ordem e a reamostragem pelo vizinho mais próximo. Aceitou-se a correção geométrica individual apenas quando o RMS apresentou valor menor que 0,5 pixel, conforme sugerido por Dai \& Khorram (1998) e Jensen (2005). 
Em seguida, as imagens Landsat 5 TM de 2000, 2005, 2008 e 2011 foram submetidas a uma classificação supervisionada. A classificação automática foi feita no software ERDAS 9.1, ferramenta Maximum Likelihood, com utilização de duas classes, floresta e não floresta. A classificação supervisionada é um dos procedimentos mais utilizados para análises quantitativas de imagens no sensoriamento remoto (Richards, 2006). A distribuição utilizada na classificação Maximum Likelihood é a normal (Novo, 2008). Isso quer dizer que objetos pertencentes à mesma classe apresentarão resposta espectral próxima à média de valores para aquela classe (Ribeiro et al., 2007). Nessa classificação cada pixel é destinado à classe que apresenta a mais alta probabilidade, isto é, a máxima verossimilhança (Meneses \& Almeida, 2012).

A imagem classificada para o ano 2008 foi submetida à análise de acurácia, com objetivo de avaliar a qualidade da classificação. Para tanto, 100 pontos foram aleatorizados na imagem Landsat 5 TM classificada. Nesse caso, os pontos aleatorizados foram avaliados no software ERDAS 9.1 a partir da ferramenta Accuracy Assessment utilizando-se como verdade de campo a imagem SPOT 5. De posse do arquivo shapefile dos limites municipais foi possível efetuar um recorte da cena classificada e em seguida realizar o cálculo das métricas por meio da ferramenta Patch Grid, extensão do programa ArcGIS 9.3. As métricas calculadas no estudo foram: índice médio de proximidade (MPI), menor distância do vizinho (MNN), total de bordas (TE), densidade de bordas (ED), tamanho médio da mancha (MPS), número de fragmentos (NUMP) e área da classe (CA). A grande vantagem do uso de métricas de paisagem é a possibilidade de obtenção de indicadores que demonstram de maneira rápida as transformações ambientais (Uuemaa et al., 2013).

De posse das métricas de paisagem para os municípios de Corumbiara e Buritis, foram realizadas comparações em relação à fragmentação da floresta nesses municípios e em relação às mudanças dos usos da terra ao longo do período 2000 a 2011.

\section{RESULTADOS E DISCUSSÃO}

O modelo foi capaz de classificar corretamente $72,73 \%$ da floresta, subestimando $27 \%$ da área de floresta nativa e superestimando $11 \%$ dos outros tipos de uso da terra (Tabela 2). O Kappa calculado atingiu $78 \%$, considerada por Landis \& Koch (1977) como uma classificação muito boa.

A maior parte dos erros de subestimação está relacionado à grande presença de nuvens na imagem, pois não foram encontradas imagens Landsat com mês/dia próximos a imagem SPOT. Assim, a imagem do ano 2008 inviabilizou a classificação em determinados pontos. Contudo, não ocorreram erros de omissão (subestimação) para outros usos da terra e nem erros de comissão (superestimação) para floresta. Portanto, o método aplicado foi conservador, apresentando boa acurácia na classificação da floresta. O classificador apresentou resultados satisfatórios com acurácia global de $91 \%$, valor acima do que está estabelecido por Jensen (1986), de 85\%.

O valor da acurácia, entretanto, diminuiria caso mais classes fossem utilizadas, pois ocorreriam mais erros do classificador. Porém, a cobertura de interesse era floresta, que apresentou ótimos valores de acertos na classificação.

Tabela 2. Acurácia da classificação.

Table 2. Classification accuracy.

\begin{tabular}{|c|c|c|c|c|c|c|}
\hline \multirow{2}{*}{\multicolumn{2}{|c|}{ Floresta }} & \multicolumn{3}{|c|}{ Imagem referência } & \multirow{2}{*}{$\begin{array}{c}\text { Acurácia do } \\
\text { produtor }\end{array}$} & \multirow{2}{*}{$\begin{array}{c}\text { Acurácia do } \\
\text { usuário }\end{array}$} \\
\hline & & Outros usos & Total & & & \\
\hline \multirow{3}{*}{ Imagem classificada } & Floresta & 24 & 0 & 24 & $72,73 \%$ & \multirow{8}{*}{$\begin{array}{c}100 \% \\
88,16 \%\end{array}$} \\
\hline & Outros usos & 9 & 67 & 76 & $100 \%$ & \\
\hline & Total & 33 & 67 & 100 & & \\
\hline \multicolumn{2}{|c|}{ Erros de omissão } & $27 \%$ & $0 \%$ & & & \\
\hline \multicolumn{2}{|c|}{ Erros de comissão } & $0 \%$ & $11 \%$ & & & \\
\hline \multicolumn{2}{|c|}{ Estatística do Kappa } & 1,0 & 0,64 & & & \\
\hline \multicolumn{2}{|c|}{ Kappa geral } & $78,1 \%$ & & & & \\
\hline \multicolumn{2}{|c|}{$\begin{array}{l}\text { Acurácia geral da } \\
\text { classificação }\end{array}$} & $91 \%$ & & & & \\
\hline
\end{tabular}




\subsection{Estudo da paisagem - município de Corumbiara}

Corumbiara apresentou desmatamento crescente da floresta nativa ao longo do período de análise. $\mathrm{O}$ desmatamento nesse município segue o mesmo padrão que pode ser observado em todo o estado de Rondônia, com clareiras que são iniciadas em áreas adjacentes às estradas principais, gerando assim o desmatamento em geometria do tipo "espinha de peixe" (Alves et al., 2003). Essa geometria de desmatamento interfere na fragmentação da floresta (Batistella et al., 2000). A classificação em floresta e não floresta para o município de Corumbiara, RO, revelou aumento da degradação da floresta nativa ao longo dos anos, a qual foi maior no período de 2000 a 2005 (Figura 2).

No município de Corumbiara ocorreu um aumento no número de fragmentos de floresta (NUMP) e ao mesmo tempo um declínio na área da classe (CA) floresta no período de análise (Tabela 3).
O tamanho médio da mancha (MPS) de floresta diminui de 2000 para 2005 devido à perda de área da classe. Nesse caso, observa-se diminuição da conectividade das manchas e o ambiente se torna mais suscetível às adversidades ambientais. $\mathrm{O}$ tamanho do maior fragmento para a classe floresta também foi alterado. Em 2000, seu tamanho era $35.567,60$ hectares, diminuindo para 33.367,46 em 2005 e para 32.563,59 hectares em 2011. Quanto menor o tamanho do remanescente, maior será a influência de fatores externos na mancha, o que faz com que os ecossistemas dos fragmentos menores sejam influenciados predominantemente por fatores externos (Saunders et al., 1991). Além disso, um fragmento maior pode conter todos os habitats que poderiam ocorrer em uma região, diferente de fragmentos menores, que abrigam poucos habitats.

Remanescentes de floresta com pequenas áreas, 0,05 a 0,10 hectares e 0,11 a 0,5 hectares (Figura 3 ) estão presentes em maior número no município de Corumbiara. Essas classes de fragmento de menor área revelam um aumento no número desses fragmentos do ano de 2000 para o ano de 2005 e uma diminuição no
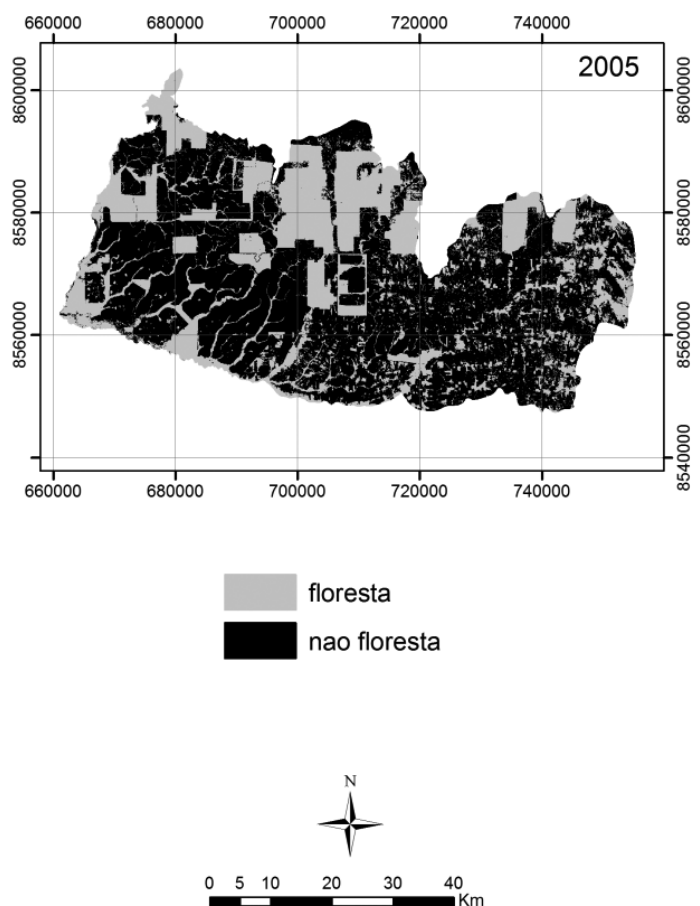

Sistema de Projeção UTM Datum SAD 69 Zona $20 \mathrm{~S}$

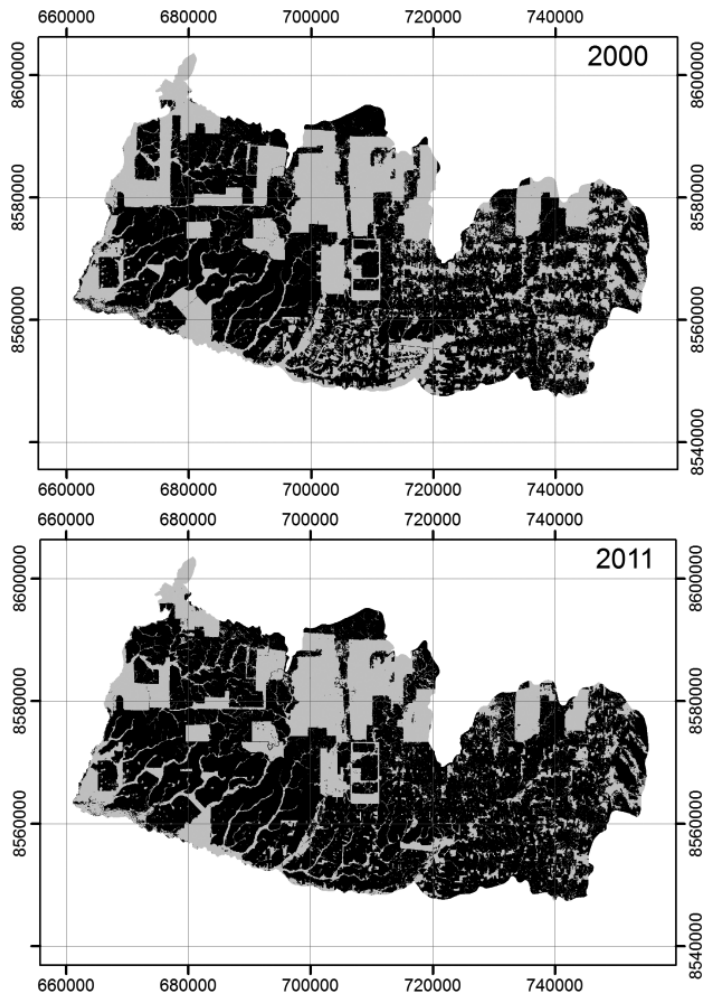

Figura 2. Classificação das áreas de floresta e não floresta do município de Corumbiara, RO nos anos 2000, 2005 e 2011. Figure 2. Forest and non-forest classification of Corumbiara at 2000, 2005 and 2011. 
Tabela 3. Métricas de análise de classe para o município de Corumbiara, RO, anos 2000, 2005 e 2011.

Table 3. Class analysis metrics to Corumbiara at 2000, 2005 and 2011.

\begin{tabular}{|ccccccccc}
\hline Classe 2000 & MPI & MNN & TE & ED & MPS & NUMP & CA \\
\hline Floresta & 25.524 & 72 & $9.294,96$ & 32,23 & 17,10 & 7.157 & 122.414 \\
\hline Não floresta & 473.536 & 68 & $9.228,09$ & 32,00 & 63,78 & 2.602 & 165.960 \\
\hline Classe 2005 & MPI & MNN & TE & ED & MPS & NUMP & CA \\
\hline Floresta & 15.815 & 77 & $9.263,04$ & 30,12 & 14,32 & 7.780 & 111.443 \\
\hline Não floresta & 723.624 & 61 & $9.231,48$ & 30,01 & 94,07 & 2.085 & 196.139 \\
Classe 2011 & MPI & MNN & TE & ED & MPS & NUMP & CA \\
\hline Floresta & 14.370 & 82 & $8.753,76$ & 28,43 & 15,20 & 6.865 & 104.340 \\
\hline Não floresta & 634.860 & 71 & $8.766,96$ & 28,47 & 82,65 & 2.463 & 203.569 \\
\hline
\end{tabular}

MPI = Índice médio de proximidade $(\mathrm{m}) ; \mathrm{MNN}=$ Menor distância do vizinho (m); TE = Total de bordas (m); ED = Densidade de bordas (\%); MPS = Tamanho médio da mancha (ha); NUMP = Número de fragmentos; CA = Área da classe (ha).

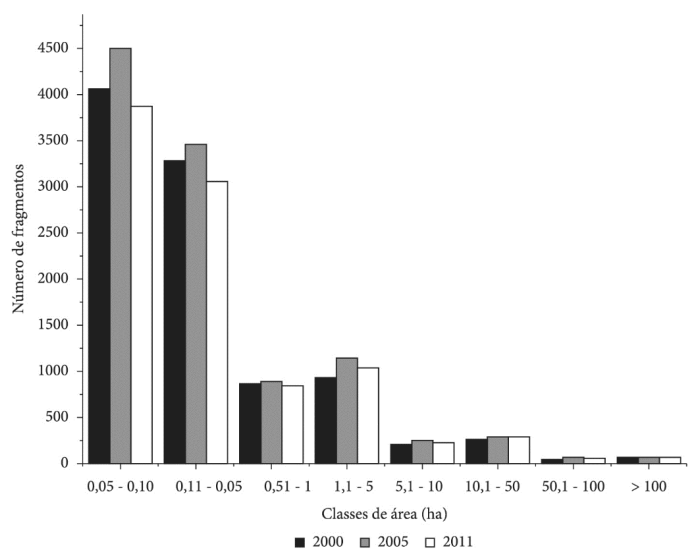

Figura 3. Número de fragmentos por classe de remanescentes nos anos 2000, 2005 e 2011 no município de Corumbiara.

Figure 3. Fragments numbers as a function of remaining classes (columns) and total area of each remaining class (rows) at 2000, 2005 and 2011 in Corumbiara.

ano de 2011 (Figura 3), que pode ter como explicação o desmatamento desses fragmentos, que passam a representar a classe não floresta.

Em contrapartida, remanescentes com áreas maiores, 50,1 a 100 hectares e $>100$ hectares (Figura 3), possuem pouca representação na área em questão, com pequena quantidade de fragmentos.

A área total da classe com fragmentos maiores que 100 hectares diminui significativamente do ano 2000 (118.042,1 hectares) para o ano 2005 (95.000,65 hectares). Entretanto, o desmatamento foi menor ao se comparar a classificação do ano 2005 à do ano 2011 (88.629,29 hectares). A fragmentação da Amazônia no ano 2006 aumentou apenas em dados relativos, mas não absolutos, pois ocorreu redução de desmatamento de grandes polígonos (acima de $100 \mathrm{ha}$ ), que representavam $40 \%$ do desmatamento total no ano 2002, com a manutenção de desmatamentos menores, de 50 ha (Brasil, 2009). Essa redução aumentou a importância dos desmatamentos menores, como é o caso dos desmatamentos ocorridos entre os anos 2000 a 2011 no município de Corumbiara. Assim, mesmo com grandes áreas desmatadas, o município de Corumbiara ainda apresenta redução das áreas de floresta. Porém essa redução da floresta nativa ocorre em proporções menores, mas que têm grande relevância em termos de impactos na área de floresta que ainda resta.

A soma das bordas da classe floresta (Tabela 3) diminuiu ao longo dos anos e uma das justificativas pode estar na diminuição da área da classe, levando à diminuição de suas bordas no geral. O mesmo pode ser descrito para a densidade de bordas (ED). Uma elevada densidade de bordas pode representar alto grau de complexidade da paisagem, visto positivamente (Lang \& Blaschke, 2007). Porém, no caso estudado sabe-se que esses valores representam um fator de fragmentação ou retalhamento do local, com consequências negativas para a conservação da biodiversidade na paisagem de estudo.

O agravamento do fator de fragmentação também pode ser observado pela diminuição do índice médio de proximidade (Tabela 3) para a classe floresta ao longo dos anos, atestando que os fragmentos da floresta se tornaram mais isolados ao longo dos anos. Para a classe não floresta ocorreu um aumento do MPI (período 2000-2005), diminuindo o isolamento das manchas de não floresta. Isso foi ocasionado pelo desmatamento de fragmentos de floresta, que passaram 
a ser inclusos na classe não floresta, conectando essas novas manchas às da classe e assim diminuindo o seu isolamento. $\mathrm{O}$ isolamento aumenta o risco de incêndios em fragmentos remanescentes de floresta, já que há aumento de sua borda, além da presença de usos que podem provocar incêndios (Cochrane et al., 2002).

A distância ao vizinho mais próximo (MNN) para a classe de floresta cresceu ao longo dos anos; em contraste, para a classe não floresta essa métrica diminuiu no período 2000-2005 e aumentou no período 2005-2011. O aumento no isolamento das manchas de floresta é previsto e coerente, devido aos processos de fragmentação e degradação que a floresta sofreu ao longo dos anos.

\subsection{Estudo da paisagem - município de Buritis}

O aumento do desmatamento em Rondônia está ligado a intensa pressão por áreas para criação de gado e cultivo de grãos (Alves et al., 2003), com massiva conversão de floresta nativa em pastos (Ferraz et al., 2006, 2009). Um exemplo disso foi o crescente desmatamento ocorrido entre os anos 2000 e 2011 no município de Buritis (Figura 4). Além disso, ressalta-se que o tamanho do maior fragmento para a classe floresta apresentou significativa redução ao longo dos anos, de $240.168,32$, em 2000, para 72.184,11 hectares, em 2005, e 30.849,87 hectares em 2011.

O tamanho do fragmento é de extrema importância, pois a riqueza de espécies declina juntamente com a área do fragmento (Laurance et al., 2011). Pequenos fragmentos não são capazes de sustentar os efeitos deletérios das bordas (Laurance et al., 2011), além de perderem espécies mais rapidamente (Stouffer et al., 2008).

O total de bordas da classe floresta aumentou entre 2000 e 2005, caindo em 2011 devido a um aumento no número de fragmentos da floresta, aumentando o número de bordas. A queda do total de bordas em 2011 é resultado da conversão desses fragmentos de floresta em não floresta. A conversão de habitats
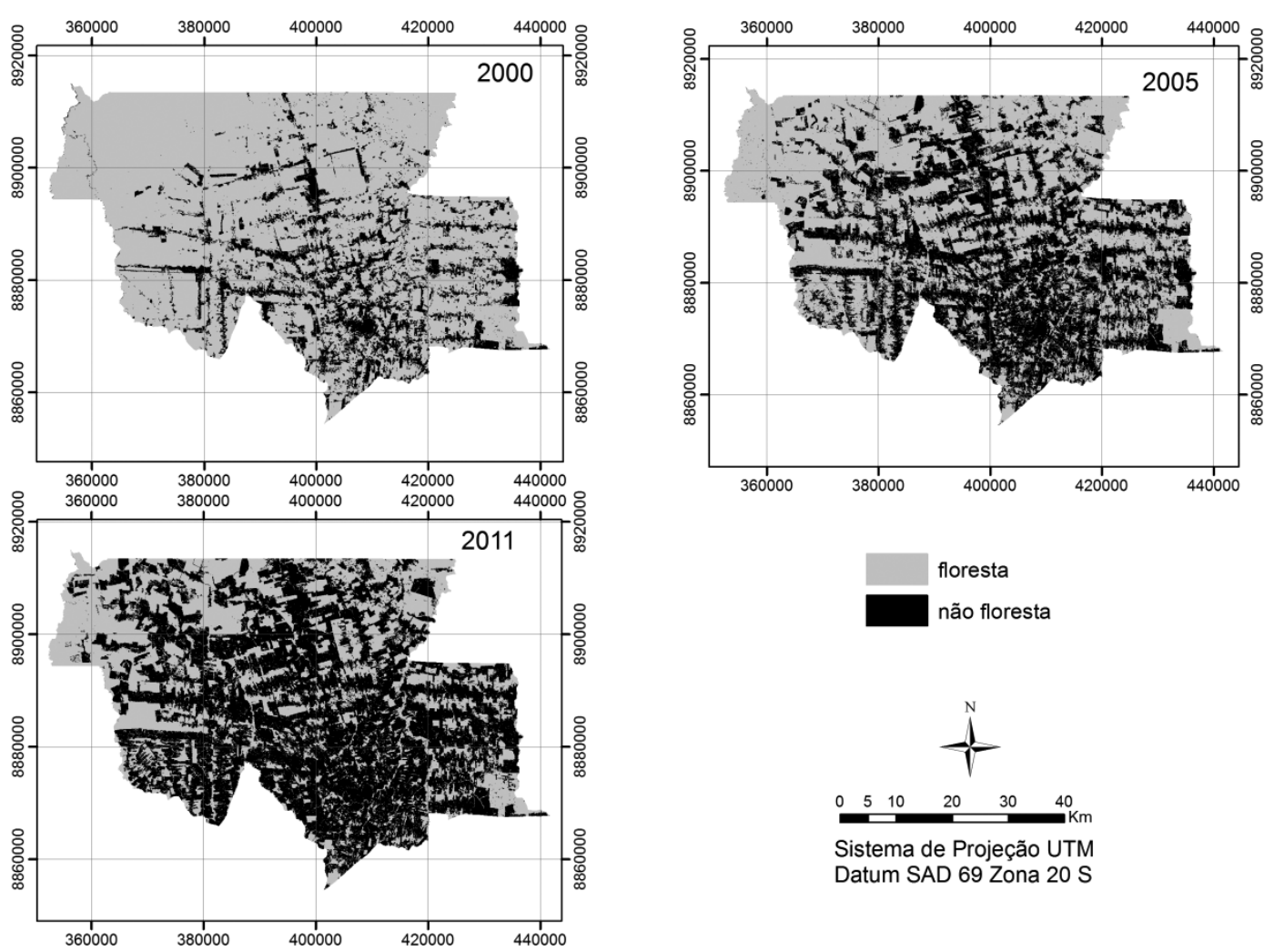

Figura 4. Classificação em floresta e não floresta para o município de Buritis, RO, nos anos 2000, 2005 e 2011. Figure 4. Forest and non-forest classification to Buritis (RO), at 2000, 2005 and 2011. 
contínuos para habitats remanescentes aumenta o tamanho da borda entre fragmentos e seus habitats em volta (Collinge, 1996), criando graves mudanças no microclima dos fragmentos remanescentes, causadas pelo efeito de borda (vento, umidade e luminosidade). A densidade de bordas segue o mesmo raciocínio que o total de bordas.

O aumento da menor distância do vizinho para a classe floresta (Tabela 4) durante a década analisada se deve aos efeitos da fragmentação e substituição de usos dessa classe, o que leva a um isolamento cada vez maior das manchas da mesma classe. $\mathrm{O}$ aumento da métrica MNN também foi observado por Ferraz et al. (2006). O mesmo raciocínio pode ser aplicado para o índice médio de proximidade, em que para a classe floresta esse índice diminuiu ao longo da década, indicando aumento no isolamento das manchas de mesma classe. Esse isolamento é prejudicial para a dispersão de plantas e movimentação de animais, podendo dificultar a conexão entre os habitats, diminuindo a riqueza e composição de espécies (Collinge, 1996).

Houve um aumento significativo no número de fragmentos com menor área ( 0,05 a 0,10 ha; 0,11 a 0,5 ha) de 2000 para 2005, com diminuição no ano de 2011 (devido à conversão dos fragmentos de floresta para outros usos) (Figura 5). Esse mesmo resultado foi observado por De Barros Ferraz et al. (2005) e Trani \& Giles (1999), que indicaram um aumento do número de fragmentos no início do processo de fragmentação, seguido por uma redução nesses fragmentos (tanto em tamanho da mancha quanto no número de manchas) nos anos que se seguem.

Fragmentos de menor área estão presentes em maior número no município de Buritis (Figura 5).
$\mathrm{O}$ aumento de pequenos fragmentos na Amazônia já foi relatado em outros estudos, como Numata \& Cochrane (2012). Esses autores relatam que no período de estudo de 2000 a 2010 observaram intensos processos de fragmentação na Amazônia em que fragmentos pequenos ( $<100$ hectares) dobraram em quantidade, introduzindo assim maior quantidade de bordas na paisagem. Grandes fragmentos diminuíram significativamente ao longo da última década (Figura 5).

As classes de remanescentes de floresta com fragmentos de menor área, 0,05-0,10 e 0,11-0,5 têm área total inferior às classes com fragmentos maiores, apresentando 1.628 hectares no ano de 2011. A classe com fragmentos maiores que 100 hectares apresenta área de 121.653,9 hectares no ano de 2011.

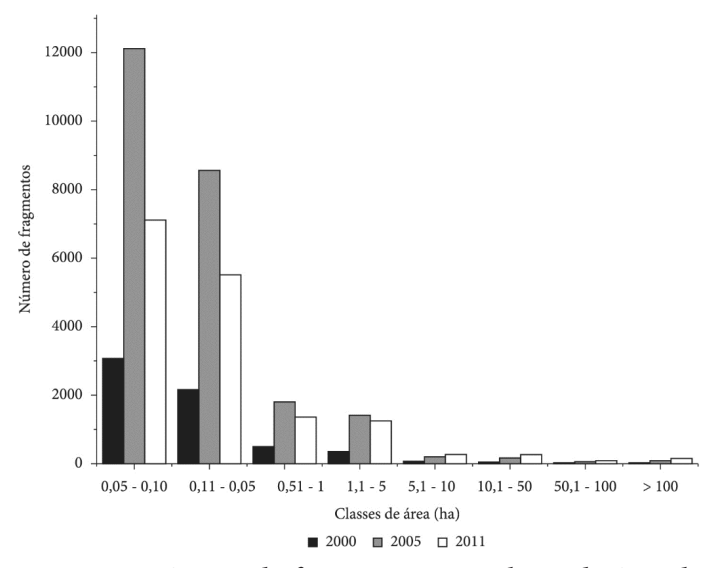

Figura 5. Número de fragmentos por classe de área de remanescentes de floresta nativa nos anos 2000, 2005 e 2011 em Buritis.

Figure 5. Fragments numbers as a function of area remaining classes at 2000, 2005 and 2011 in Buritis.

Tabela 4. Métricas de análise de classe para o município de Buritis, RO, anos 2000, 2005 e 2011.

Table 4. Class analysis metrics to Buritis - RO, at 2000, 2005 and 2011.

\begin{tabular}{cccccccc} 
Classe 2000 & MPI & MNN & TE & ED & MPS & NUMP & CA \\
\hline Floresta & $1.181 .544,40$ & 47,68 & 943.622 & 31,04 & 65,55 & 3.823 & $250.589,57$ \\
\hline Não floresta & $10.849,65$ & 84,41 & 91.763 & 30,19 & 7,50 & 7.114 & $53.388,11$ \\
\hline Classe 2005 & MPI & MNN & TE & ED & MPS & NUMP & CA \\
\hline Floresta & $135.108,48$ & 50,29 & 187.456 & 57,83 & 12,53 & 15.388 & $192.794,85$ \\
\hline Não floresta & $230.981,56$ & 61,37 & 184.979 & 57,06 & 9,44 & 13.912 & $131.379,03$ \\
Classe 2011 & MPI & MNN & TE & ED & MPS & NUMP & CA \\
\hline Floresta & $20.191,42$ & 68,70 & 133.408 & 41,18 & 12,25 & 11.375 & $139.354,11$ \\
\hline Não floresta & $437.020,92$ & 63,01 & 131.576 & 40,61 & 39,99 & 4.617 & $184.630,77$ \\
\hline
\end{tabular}

MPI = Índice médio de proximidade (m); MNN = Menor distância do vizinho (m); TE = Total de bordas (m); ED = Densidade de bordas (\%); MPS = Tamanho médio da mancha (ha); NUMP = Número de fragmentos; CA = Área da classe (ha). 


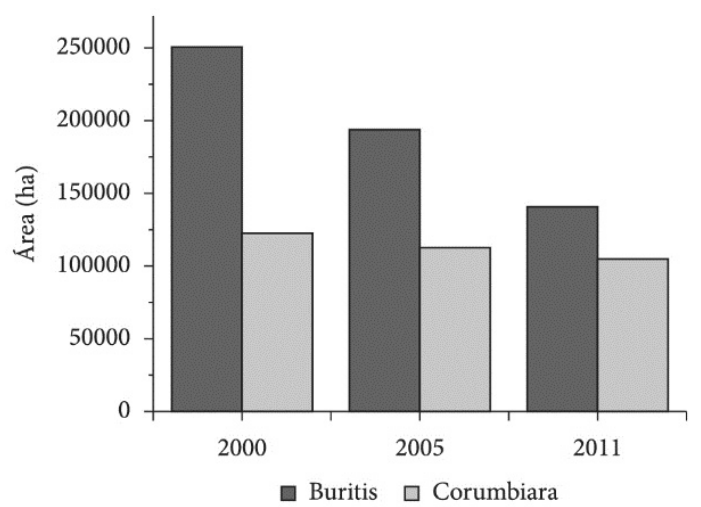

Figura 6. Perda de floresta nativa nos municípios de Corumbiara e Buritis nos anos 2000, 2005 e 2011.

Figure 6. Corumbiara and Buritis native forest losses at 2000, 2005 and 2011.

A perda de floresta foi maior para o município de Buritis, que tem sua ocupação mais recente (Figura 6). Corumbiara já passou por esse processo na década anterior, porém ainda mantém a perda de floresta nativa. Se não forem adotadas medidas de conservação, o município de Buritis provavelmente terá grande parte de sua floresta nativa degradada, como ocorreu em Corumbiara.

\section{CONCLUSÕES}

A análise das métricas de paisagem (MPI, MNN, TE, ED, MPS, NUMP e CA) permitiu avaliar a fragmentação e perda da floresta nativa dos municípios de Corumbiara e Buritis. Os resultados obtidos apontam para o aumento contínuo da fragmentação da floresta nativa, comprovado pelo aumento no número de fragmentos dessa classe e diminuição da conectividade dos fragmentos. Além disso, observou-se um crescente desmatamento ao longo da última década, com grande perda de área da classe floresta. No município de Corumbiara, apesar da perda da maior parte de sua cobertura natural, entretanto, continuam intensos os processos de degradação e fragmentação da floresta. Em contraste, Buritis apresentou valores mais altos de perda florestal e fragmentação, com aumento dos fragmentos de floresta em proporções maiores do que em Corumbiara. Isso se deve ao fato de Buritis representar uma nova fronteira de ocupação no estado de Rondônia.
Análises futuras poderiam incluir maior série temporal, incluindo a aplicação de outras métricas de paisagem, como as de área central e/ou de forma, avaliando mais detalhadamente os processos de fragmentação desses municípios.

\section{STATUS DA SUBMISSÃO}

Recebido: 23 maio 2013

Aceito: 17 fev., 2015

\section{AUTOR(ES) PARA CORRESPONDÊNCIA}

\section{Eraldo Aparecido Trondoli Matricardi}

Departamento de Engenharia Florestal, Universidade de Brasília - UnB, Brasília, DF, Brasil e-mail: ematricardi@gmail.com

\section{REFERÊNCIAS}

Alves DS, Escada MIS, Pereira JLG, de Albuquerque Linhares CA. Land use intensification and abandonment in Rondônia, Brazilian Amazônia. International Journal of Remote Sensing 2003; 24(4): 899-903. http://dx.doi.or g/10.1080/0143116021000015807.

Arima EY, Walker RT, Sales M, Souza C Jr, Perz SG. The fragmentation of space in the Amazon basin: emergent road networks. Photogrammetric Engineering and Remote Sensing 2008; 74(6): 699-709. http://dx.doi.org/10.14358/ PERS.74.6.699.

Batistella M, Brondizio ES, Moran EF. Comparative analysis of landscape fragmentation in Rondônia, Brazilian Amazon. International Archives of Photogrammetry and Remote Sensing 2000; XXXIII(B7): 148-155.

Becker BK, Miranda M, Machado LO. Fronteira Amazônica: questões sobre a gestão do território. Brasília, DF: Editora UnB; 1990.

Brasil. Ministério do Meio Ambiente. Plano de prevenção e controle do desmatamento na Amazônia Legal - PPCDAM. Brasília: Ministério do Meio Ambiente; 2009.

Briant G, Gond V, Laurance SG. Habitat fragmentation and the desiccation of forest canopies: a case study from eastern Amazonia. Biological Conservation 2010; 143(11): 2763-2769. http://dx.doi.org/10.1016/j.biocon.2010.07.024.

Broadbent EN, Asner GP, Keller M, Knapp DE, Oliveira JC, Silva JN. Forest fragmentation and edge effects from deforestation and selective logging in the Brazilian Amazon. Biological Conservation 2008; 141(7): 1745-1757. http:// dx.doi.org/10.1016/j.biocon.2008.04.024. 
Bunker SG. Underdeveloping the Amazon: extraction, unequal exchange, and the failure of the mordern State. USA: University of Chicago Press; 1999.

Chander G, Markham B, Helder D. Summary of current radiometric calibration coefficients for Landsat MSS, TM, ETM+ and EO-1 ALI sensors. Remote Sensing of Environment 2009; 113(5): 893-903. http://dx.doi. org/10.1016/j.rse.2009.01.007.

Cochrane MA, Laurance WF. Fire as a large-scale edge effect in Amazonian Forests. Journal of Tropical Ecology 2002; 18(03): 311-325. http://dx.doi.org/10.1017/ S0266467402002237.

Cochrane MA, Skole DL, Matricardi EAT, Chomentowski WH. Interaction and sinerg between selective logging, forest fragmentation and fire disturbance in tropical florests: case study Mato Grosso, Brazil. Michigan, USA: CGCEO; 2002.

Cohen AD. A coefficient of agreement for nominal scales. Educational and Psychological Measurement 1960; 20(1): 37-46. http://dx.doi.org/10.1177/001316446002000104.

Collinge SK. Ecological consequences of habitat fragmentation: implications for landscape architecture and planning. Landscape and Urban Planning 1996; 36(1): 59-77. http:// dx.doi.org/10.1016/S0169-2046(96)00341-6.

Colson F, Bogaert J, Carneiro A Fo, Nelson B, Pinagé $\mathrm{ER}$, Ceulemans R. The influence of forest definition on landscape fragmentation assessment in Rondônia, Brazil. Ecological Indicators 2009; 9(6): 1163-1168. http://dx.doi. org/10.1016/j.ecolind.2009.02.001.

Companhia Nacional de Abastecimento - CONAB. Séries históricas de área plantada por unidades da federação de 1976 a 2011 [online]. [citado 2012 abr. 15]. Disponível em: http://www.conab.gov.br/OlalaCMS/uploads/ arquivos/15_06_11_10_17_04_brasilufseriehist.xls

Congalton RG. A review af assessing the accuracy of classifications of remotely sensed data. Remote Sensing of Environment 1991; 37(1): 35-46. http://dx.doi. org/10.1016/0034-4257(91)90048-B.

Dai X, Khorram S. The effects of image misregistration on the accuracy of remotely sensed change detection. IEEE Transactions on Geoscience and Remote Sensing 1998; 36(5): 1566-1577. http://dx.doi.org/10.1109/36.718860.

De Barros Ferraz SFB, Vettorazzi CA, Theobald DM, Ballester MVR. Landscape dynamics of Amazonian deforestation between 1984 and 2002 in central Rondônia, Brazil: assessment and future scenarios. Forest Ecology and Management 2005; 204(1): 69-85. http://dx.doi. org/10.1016/j.foreco.2004.07.073.

Fearnside PM. Soybean cultivation as a threat to the environment in Brazil. Environmental Conservation 2001; 28(1): 23-28. http://dx.doi.org/10.1017/S0376892901000030.

Ferraz SFB, Capão LMSAC, Vettorazzi CA. Temporal scale and spatial resolution effects on Amazon forest fragmentation assessment in Rondônia. International
Journal of Remote Sensing 2006; 27(3): 459-472. http:// dx.doi.org/10.1080/01431160500259907.

Ferraz SFB, Vettorazzi CA, Theobald DM. Using indicators of deforestation and land-use dynamics to support conservation strategies: a case study of central Rondônia, Brazil. Forest Ecology and Management 2009; 257(7): 1586-1595. http:// dx.doi.org/10.1016/j.foreco.2009.01.013.

Instituto Nacional de Pesquisas Espaciais - INPE. Projeto PRODES - monitoramento da floresta amazônica por satélite [online]. [citado 2015 jan. 19]. Disponível em: http://www.obt.inpe.br/prodes/sisprodes2000_2014.htm

Jensen JR. Introductory digital image processing. Englewood Cliffs: Prentice Hall; 1986.

Jensen JR. Introductory digital image processing: a remote sensing perspective. Upper Saddle River: Pearson Prentice Hal; 2005.

Landis JR, Koch GG. The measurement of observer agreement for categorical data. Biometrics 1977; 33(1): 159-174. http:// dx.doi.org/10.2307/2529310. PMid:843571.

Lang S, Blaschke T. Análise da paisagem com SIG. São Paulo: Oficina de Textos; 2007.

Laurance WF, Camargo JLC, Luizão RCC, Laurance SG, Pimm SL, Bruna EM et al. The fate of Amazonian forest fragments: a 32-year investigation. Biological Conservation 2011; 144(1): 56-67. http://dx.doi.org/10.1016/j. biocon.2010.09.021.

Laurance WF, Curran TJ. Impacts of wind disturbance on fragmented tropical forests: a review and synthesis. Austral Ecology 2008; 33(4): 399-408. http://dx.doi. org/10.1111/j.1442-9993.2008.01895.x.

Malingreau JP, Tucker CJ. Large-scale deforestation in the southeastern Amazon basin of Brasil. Ambio 1988; 17(1): 49-55.

Matos JM, Rocha VMDAS, Carvalho APFDE. Avaliação da evolução do desmatamento em assentamentos do Incra a partir dos dados do Prodes e Deter para os anos 1997 2010. In: Anais do XV Simpósio Brasileiro de Sensoriamento Remoto - SBSR; 2011; Curitiba, PR. Curitiba, PR: INPE; 2011. p. 5833-5840.

Meneses PR, Almeida TDE. Introdução ao processamento de imagens de sensoriamento remoto. Brasília, DF: CNPQ; 2012.

Nepstad D, McGrath D, Alencar A, Barros AC, Carvalho $\mathrm{G}$, Santilli $\mathrm{M}$ et al. Environment: frontier governance in Amazonia. Science 2002; 295(5555): 629-631. http://dx.doi. org/10.1126/science.1067053. PMid:11809955.

Novo EMLM. Sensoriamento remoto: princípios e aplicações. 3. ed. São Paulo: Blucher; 2008.

Numata I, Cochrane MA. Forest fragmentation and its potential implications in the Brazilian Amazon between 2001 and 2010. Open Journal of Forestry 2012; 2(4): 265 271. http://dx.doi.org/10.4236/ojf.2012.24033. 
Pedlowski MA, Dale VH, Matricardi EAT, Silva EP Fo. Patterns and impacts of deforestation in Rondonia, Brazil. Landscape and Urban Planning 1997; 38(3-4): 149-157. http://dx.doi.org/10.1016/S0169-2046(97)00030-3.

Perdigão F, Bassegio L. Migrantes Amazônicos, Rondônia: a tragetória da ilusão. São Paulo: Loyola; 1992.

Ribeiro EJC, Baptista GMM, Bias ES. Comparação dos métodos de classificação supervisionada de imagem máxima verossimilhança e redes neurais em ambiente urbano. In: Anais do XIII Simpósio Brasileiro de Sensoriamento Remoto; 2007; Florianopólis. Florianopólis: INPE; 2007. p. 5471-5478.

Richards JA. Remote sensing digital image analysis: an introduction. Australia: Springer - Verlag berlin Heidelberg; 2006.

Saunders DA, Hobbs RJ, Margules CR. Biological consequences of ecosystem fragmentation: a review. Conservation Biology 1991; 5(1): 18-32. http://dx.doi. org/10.1111/j.1523-1739.1991.tb00384.x.

Schmink M, Wood CH. Contested frontiers in Amazonia. New York: Columbia University Press; 1992.

Shida CN, Pivello VR. O contexto do Cerrado Pé-deGigante como fragmento: consequências. o desafio da conservação dos recursos naturais do Cerrado Pé-deGigante. In: Pivello VR, Varanda EM, editores. O cerrado
Pé-de-Gigante - Parque Estadual de Vassununga. São Paulo: SMA; 2005.

Skole D, Tucker C. Tropical deforestation and habitat fragmentation in the Amazon: satellite data from 1978 to 1988. Science 1993; 260(5116): 1905-1910. http://dx.doi. org/10.1126/science.260.5116.1905. PMid:17836720.

Stouffer PC, Strong C, Naka LN. Twenty years of understory bird extinctions from Amazonian rain forest fragments: consistent trends and landscape-mediated dynamics. Diversity \& Distributions 2009; 15(1): 88-97. http://dx.doi. org/10.1111/j.1472-4642.2008.00497.x.

Trani MK, Giles RH Jr. An analysis of deforestation: metrics used to describe pattern change. Forest Ecology and Management 1999; 114(2-3): 459-470. http://dx.doi. org/10.1016/S0378-1127(98)00375-2.

U.S. Department of the Interior. U.S. Geological survey - USGS [online]. [citado 2012 jun. 15]. Disponível em: http://earthexplorer.usgs.gov/

University of Maryland. Global land cover facility [online]. [citado 2012 jun. 20]. Disponível em: http://glcf.umd. edu/data/

Uuemaa E, Mander U, Marja R. Trends in the use of landscape spatial metrics as landscape indicators: a review. Ecological Indicators 2013; 28: 100-106. http://dx.doi. org/10.1016/j.ecolind.2012.07.018. 